\title{
Leishmaniasis mucocutánea en un paciente con infección por VIH
}

\author{
Juan J. Montenegro-Idrogo, Raúl A. Montañez-Valverde, Cesar Chian y Carlos Benites-Villafane
}

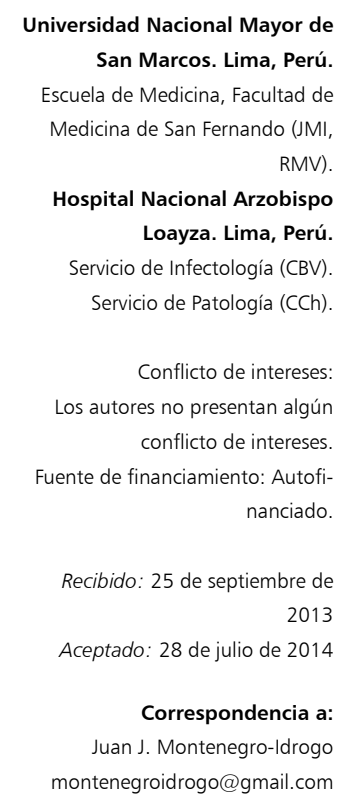

\section{Introducción}

\section{L} a leishmaniasis es una enfermedad metaxénica andino-tropical. Es considerada endémica en Latinoamérica $^{1,2}$. La forma mucocutánea, que produce una grave destrucción inflamatoria de la mucosa, es menos frecuente y se concentra principalmente en Perú, Bolivia y Brasil ${ }^{2}$.

En Perú, la incidencia acumulada de leishmaniasis en el año 2003 fue de 23,6 casos por 100.000 habitantes; sin embargo, la forma mucocutánea sólo fue de 1,2 casos por 100.000 habitantes $^{3}$. El número de casos en Perú, según la OMS, en 2011 y 2012 fue de 11.204 y 7.527, respectivamente ${ }^{4}$. En el año 2009 se confirmaron 6.172 casos, de los cuales sólo 5,4\% correspondieron a la forma mucocutánea, siendo Cusco, Loreto y Madre de Dios los lugares de procedencia más frecuentes ${ }^{5}$.

La forma mucocutánea se presenta meses o años después de la presentación y cicatrización de la forma cutánea, dependiendo de la inmunidad del hospedero, el tratamiento recibido y la especie de Leishmania ${ }^{6,7}$. Se describe incluso hasta 30 años posterior a la presentación cutánea.

Afecta preferentemente la mucosa nasal a nivel del septum cartilaginoso ${ }^{8}$ y paladar blando ${ }^{9,10}$. Posteriormente progresa y se profundiza hasta todo el paladar, pilares, úvula y faringe. Esta forma se presenta histológicamente, por lo general, como una reacción granulomatosa por una respuesta mediada por células CD4. En el caso de co-infección por VIH la respuesta inflamatoria suele ser menor, por lo que puede no encontrarse la reacción granulomatosa en las lesiones ${ }^{11,12}$. La leishmaniasis mu- cocutánea se ha asociado a una progresión de la infección por VIH y a una disminución de la expectativa de vida ${ }^{4}$. Se menciona además, que esta forma está relacionada a una respuesta inflamatoria mediada por receptores tipo TLR3 (Toll like receptor tipo 3) relacionada a un virus ARN, LRV (Leishmania RNA virus) ${ }^{13,14}$.

La leishmaniasis puede presentarse en co-infección con los virus HTLV-1 ${ }^{15}$ y VIH. En Europa, estados de inmunosupresión se han asociado preferentemente a la forma visceral ${ }^{16}$; sin embargo, en pacientes con infección por VIH se han reportado diversas formas de leishmaniasis ${ }^{17-21}$. En Perú, la infección por VIH se encuentra en el nivel de epidemia concentrada, y a pesar de haber disminuido el número de casos reportados, la prevalencia se ha mantenido constante ${ }^{7}$. Asimismo, en Latinoamérica se han comunicado casos de co-infección de leishmaniasis mucocutánea y VIH en Bolivia en $2009^{22}$ y en Perú en $1993^{23}$

Describimos un caso de leishmaniasis mucocutánea en un paciente infectado con VIH.

\section{Caso clínico}

Varón de 42 años de edad, con diagnóstico de infección por VIH desde hacía ocho años atrás, con controles inmunológicos periódicos, sin criterios para inicio de tratamiento antirretroviral. Tenía antecedentes de una leishmaniasis cutánea en el antebrazo derecho 20 años atrás, cuando residía en Madre de Dios (Amazonía oriental del Perú). Refirió que había recibido tratamiento incompleto, que no pudo especificar. Además había sido 


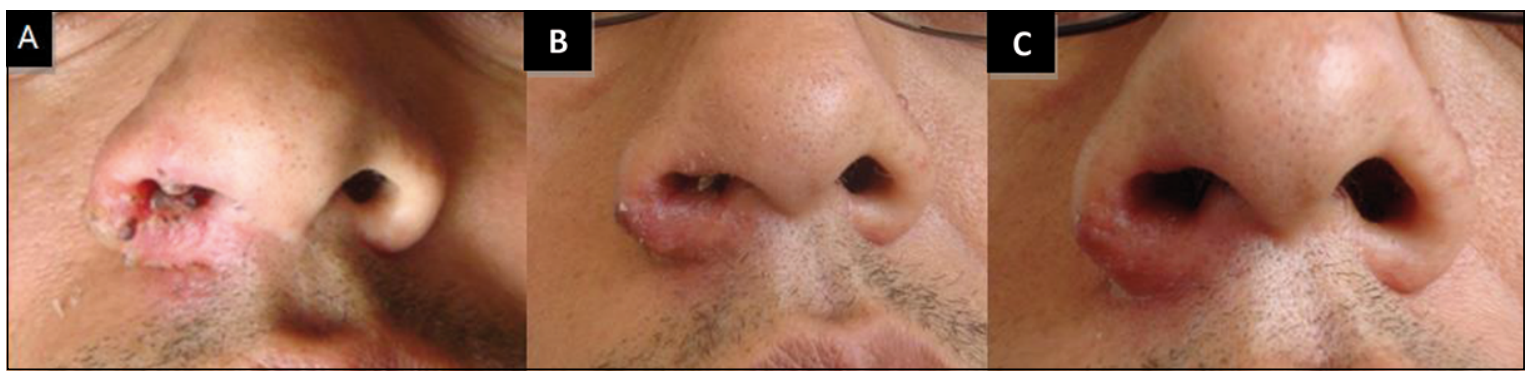

Figura 1. Lesiones pápuloulcerativas de $0,5 \mathrm{~cm}$ de diámetro en región vestibular y mucosa de la fosa nasal derecha compatibles con leishmaniasis mucocutánea: inicio (A), a las dos semanas (B) y a las 4 semanas (C) de tratamiento.

operado de un colesteatoma de oído derecho 10 años atrás, con una parálisis facial periférica derecha como secuela. No tenía antecedentes de otras enfermedades.

Tenía controles clínicos anuales de carga viral (CV) y niveles de linfocitos T CD4, manteniéndose en valores estables $\left(\mathrm{CV}<20.000\right.$ copias $/ \mathrm{ml}$ y CD4 $\left.>700 \mathrm{cél} / \mathrm{mm}^{3}\right)$, hasta que en los últimos tres meses se evidenció una leve disminución de CD4 hasta 643 (28\%) cél $/ \mathrm{mm}^{3}$ y un aumento importante de la CV hasta 47.466 copias $/ \mathrm{ml}$. En ese período tuvo episodios intercurrentes de diarrea y coriza autolimitados. Tenía además marcadores para hepatitis B positivos: HBsAg y anticore total, sin alteración de las pruebas de función hepática.

Acudió a control en el Servicio de Infectología refiriendo cefalea parietal y congestión nasal persistente, sin lesiones en el examen de nasofaringe. Un mes después presentó epistaxis, secreción nasal mucopurulenta y se observó una tumoración en la fosa nasal derecha (ala nasal) (Figura 1A).

Al examen se evidenciaron lesiones pápulo-ulcerativas de aproximadamente $0,5 \mathrm{~cm}$ de diámetro en la región vestibular y mucosa de la fosa nasal derecha, presencia de secreción mucopurulenta y una masa exofítica. Se realizó una biopsia de la tumoración nasal para estudio (Figura 3A y B).

Al examen físico presentaba además una cicatriz deprimida, delgada, lisa, hipopigmentada, de bordes irregulares de $2 \mathrm{~cm}$ de diámetro en el antebrazo derecho, correspondiente a una lesión antigua de leishmaniasis cutánea (Figura 2). Se inició tratamiento antirretroviral de gran actividad (TARGA) con zidovudina, lamivudina, lopinavir y ritonavir, ante la aparición de síntomas y sospecha de neoplasia relacionada al VIH.

El estudio histológico mostró estructuras ovaladas, de 2 a $4 \mu \mathrm{m}$ de diámetro, de tamaño uniforme, con un cinetoplasto periférico, localizadas en el citoplasma de los histiocitos, compatible con amastigotes de Leishmania spp (Figura 4A y B). La inmunofluorescencia indirecta (IFI) (con antígeno total para L. boliviana y L. brasiliensis) detectó anticuerpos anti-Leishmania a una dilución de 1/80, confirmándose el diagnóstico de leishmaniasis. Al mes siguiente de iniciado el TARGA inició tratamiento con estibogluconato sódico, $20 \mathrm{mg} / \mathrm{kg} /$ día por 30 días.

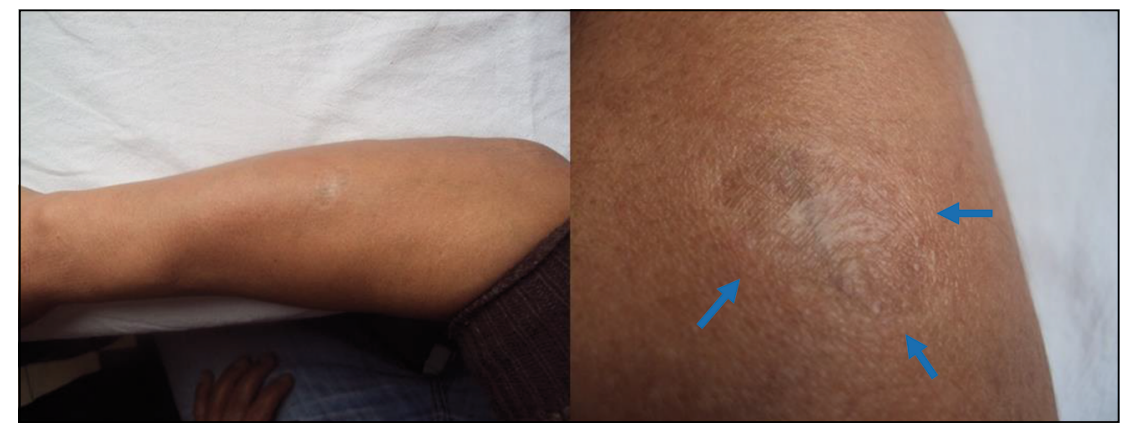

Figura 2. Cicatriz deprimida, delgada, lisa, hipopigmentada y de bordes irregulares de $2 \mathrm{~cm}$ de diámetro en antebrazo derecho, correspondiente a lesión antigua de la forma cutánea.

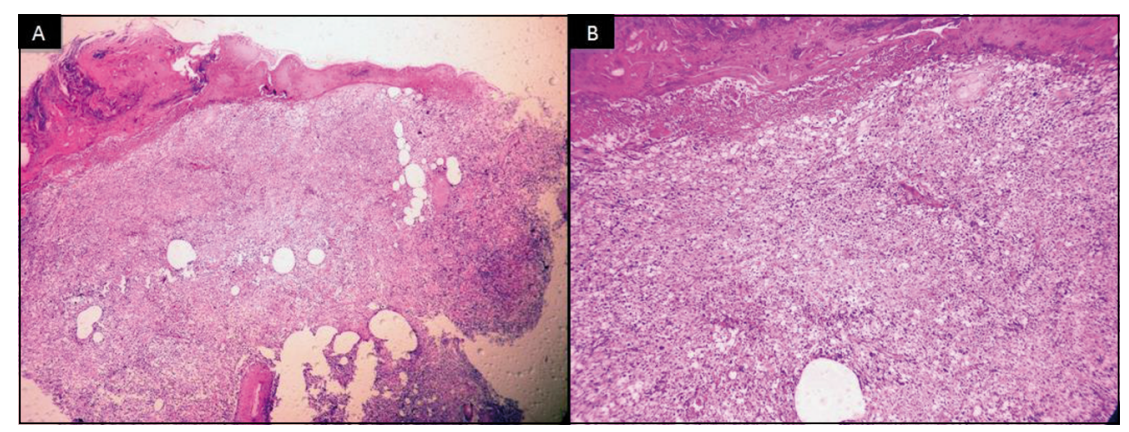

Figura 3. Biopsia de úlcera de mucosa nasal, tinción hematoxilina-eosina. A. Costra superficial y denso infiltrado inflamatorio que compromete la dermis superficial y profunda, hasta la hipodermis (10x). B. UIceración epidérmica e infiltrado inflamatorio compuesto por células de citoplasma amplio, de aspecto histiocítico. No se observan granulomas ni células gigantes multinucleadas (anergia cutánea). (40x).

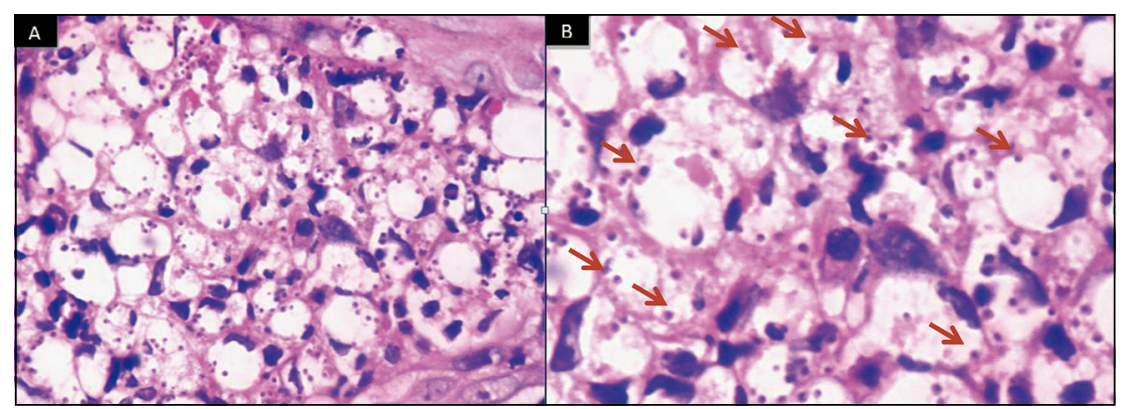

Figura 4. A. Gran cantidad de estructuras ovaladas, de 2 a $4 \mu \mathrm{m}$ de diámetro, de tamaño uniforme, con un cinetoplasto periférico, localizadas en el citoplasma de los histiocitos. (100x) B. Detalle con mayor aumento demostrando las características de los amastigotes de Leishmania. 
Luego de completar el tratamiento con estibogluconato sódico, la evolución fue favorable y la lesión mejoró entre las dos y cuatro semanas, hasta su cicatrización completa (Figura 1B y 1C). Tres meses después, los valores de CD4 se elevaron hasta 843 cél $/ \mathrm{ml}$ y la carga viral disminuyó a $<40$ copias $/ \mathrm{ml}$. Actualmente se encuentra asintomático, con TARGA, sin presentar recaída de la lesión ni lesiones similares.

\section{Discusión}

Se presenta el caso de un paciente con el antecedente de leishmaniasis cutánea 20 años atrás adquirido en Madre de Dios, Amazonía del Perú. La distribución de leishmaniasis en Perú es más frecuente en regiones de valles interandinos y selva central. Las regiones más frecuentes son Cusco (31,8\%), Loreto (19\%) y Madre de Dios $(14,2 \%)^{5,24}$.

La forma de presentación de leishmaniasis mucocutánea representa sólo $5,4 \%$ del total de $\operatorname{casos}^{5}$. La forma mucocutánea suele relacionarse con estados de inmunosupresión (neoplasias, enfermedades crónicas) e infecciones virales como el $\mathrm{VIH}^{6,7,17}$. Nuestro paciente presentaba una infección por VIH ocho años antes de la presentación clínica, sin embargo, sus niveles de CD4 se mantuvieron constantes durante todo ese período, sin presentar criterios de síndrome de reconstitución inmu$n \mathrm{e}^{25}$. Además, el cuadro sintomatológico era leve por lo que en ese momento no ameritó terapia antirretroviral.

Las manifestaciones clínicas de la leishmaniasis y su evolución a diferentes formas están determinadas por el balance entre el parásito y la inmunidad del hospedero ${ }^{8,11-14}$. Una revisión retrospectiva de 15 pacientes con leishmaniasis tegumentaria y SIDA en Brasil, encontró signos atípicos como pápulas, placas, nódulos y úlceras, algunas ubicadas en genitales ${ }^{26}$. Nuestro caso presentó signos típicos precoces de leishmaniasis mucocutánea como congestión nasal y secreción acuosa ${ }^{8,27}$. La aparición de epistaxis, secreción mucopurulenta y la presencia de una tumoración en fosa nasal derecha, se corresponden con la evolución clínica de la enfermedad, con una discreta secreción nasal inicial, similar a una rinitis, e inflamación posterior de la mucosa que se torna eritematosa, edematosa y dolorosa, que puede impresionar como tumefacción local ${ }^{4,28}$.

Dentro de los diagnósticos diferenciales están la rinitis crónica y la poliposis nasal. Por esto, es importante la búsqueda de antecedentes, ya que suele confundirse este tipo de cuadros con leishmaniasis ${ }^{29,30}$. Además se describen manifestaciones cutáneas difusas asociadas a $\mathrm{VIH}^{20,21}$.

Cerca de $90 \%$ de los cuadros de leishmaniasis mucocutánea tienen una cicatriz cutánea debido a un episodio anterior de leishmaniasis cutánea ${ }^{27}$. Lesiones desarrolladas en lugares distantes de la lesión primaria curada después de abandonado el área endémica representan evidencia indiscutible de latencia, diseminación hematógena y reactivación ${ }^{6}$, lo que ocurrió en nuestro paciente. El estadio quiescente o latente puede derivar en una reactivación de la enfermedad incluso luego de 20 ó 30 años después de la presentación inicial ${ }^{8}$. El único caso reportado anteriormente en Perú presentaba un cuadro sintomático similar y con antecedente de transmisión en la selva ${ }^{23}$, sin embargo, el tiempo de latencia en nuestro paciente fue de 20 años.

Según un estudio sobre leishmaniasis cutánea americana, la recurrencia está influenciada por la especie de Leishmania, el tratamiento recibido previamente y el estado inmunológico del paciente ${ }^{6}$. En los pacientes no tratados, cerca de $15 \%$ progresarán a la forma mucocutánea ${ }^{30}$. Por otro lado, un estudio en Sudamérica concluyó que dosis menores a $20 \mathrm{mg} / \mathrm{kg} /$ día de estibogluconato o menos de 28 días de tratamiento es un tratamiento subóptimo de leishmaniasis mucocutánea ${ }^{31}$. El paciente no terminó su primer tratamiento, lo que probablemente elevó el riesgo de reactivación, entendiendo a la leishmaniasis mucocutánea como tal.

Nuestro caso recibió tratamiento con estibogluconato $20 \mathrm{mg} / \mathrm{kg} /$ día por 30 días, considerada la terapia de elección como antimonio pentavalente ${ }^{32}$. En Brasil se utiliza el meglumine, y en Perú y Panamá los estudios existentes se realizaron con estibogluconato ${ }^{33}$. Una revisión Cochrane para Latinoamérica evaluó la eficacia según el tipo de agente específico ${ }^{34}$. Asimismo, un tratamiento completo con $20 \mathrm{mg} / \mathrm{kg} /$ día durante 20 días o más con estibogluconato, hacen muy poco probable la posibilidad de reactivación ${ }^{6}$. Actualmente el paciente se encuentra estable, sin regresión de la lesión, con terapia antirretroviral y sin otras molestias asociadas.

Una de las limitaciones de nuestro caso fue la falta de estudio microbiológico de la cepa respectiva, sin embargo, según el área de infección primaria podría sospecharse de los tipos de Leishmania (Vannia) brasiliens ${ }^{5}$. Si bien la coinfección de leishmaniasis mucocutánea y VIH no es frecuente, es importante considerar este diagnóstico en pacientes con VIH y tumoraciones de la fosa nasal, en especial de aquellos que provengan de zonas endémicas y tengan el antecedente de la forma cutánea. Asimismo, el desarrollo florido de la leishmaniasis mucocutánea no se desarrolla en todos los pacientes con la coinfección. En nuestro paciente probablemente debido a la estabilidad inmunológica en que se encontraba y el TARGA que redujo su carga viral.

\section{Resumen}

La leishmaniasis es una enfermedad metaxénica andino-tropical, considerada endémica en Perú. Su forma mucocutánea es poco frecuente. Puede presentarse en 
coinfección con los virus HTLV-1 y VIH. Se describe un caso de leishmaniasis mucocutánea en un paciente infectado con VIH, con antecedente de leishmaniasis cutánea con tratamiento incompleto 20 años atrás. Es tratado con estibogluconato sódico por 30 días, con adecuada respuesta y regresión de la lesión a las cuatro semanas. La coinfección de leishmaniasis mucocutánea y VIH no es frecuente. Las manifestaciones de leishmaniasis pueden no presentarse de forma típica en pacientes con VIH. Se debe considerar la procedencia de la zona endémica y/o el antecedente de haber presentado la forma cutánea previamente.

\section{Referencias bibliográficas}

1.- Nakkash-Chmaisse H, Makki R, Nahhas G, Knio K, Nuwayri-Salti N. Detection of Leishmania parasites in the blood of patients with isolated cutaneous leishmaniasis. Int J Infect Dis 2011; 15: e491-4.

2.- WHO. Leishmaniasis. WHO Updated January 2014. Disponible en: http://www.who.int/ mediacentre/factsheets/fs375/en/. (Consultado: junio 2014).

3.- Sánchez-Saldaña L, Sáenz-Anduaga E, Pancorbo-Mendoza J, Zegarra-Del-Carpio R, Garcés-Velasco N, Regis-Roggero A. Leishmaniasis. Dermatol Peru 2004; 14: 82-98.

4.- WHO. Leishmaniasis: Number of cases of cutaneous leishmaniasis reported Data by country. 2014 Disponible en: http://apps.who.int/gho/data/node.main. NTDLEISHCNUM?lang=en. (Consultado: junio 2014).

5.- MINSA-DGE. Análisis de la situación de salud en el Perú. Lima: MINSA; 2010. Disponible en: http://apps.who.int/gho/data/node.main. NTDLEISHCNUM?lang=en. (Consultado: junio 2014).

6.- Weigle K, Saravia NG. Natural history, clinical evolution and the host-parasite interaction in the New World cutaneous leishmaniasis. Clin Dermatol 1996; 14: 433-50.

7.- WHO. Leishmaniasis: worldwide epidemiological and drug access update. WHO 2014. Disponible en: http://www.who. int/leishmaniasis/resources/Leishmaniasis_ worldwide_epidemiological_and_drug_access_ update.pdf (Consultado: junio 2014).

8.- Goto H, Lindoso J A. Current diagnosis and treatment of cutaneous and mucocutaneous leishmaniasis. Expert Rev Anti Infect Ther 2010; 8: 419-33.

9.- de Paulo L F, Rocha G F, Luisi C M Jr, Rosa R R, Durighetto A F Jr. Mucocutaneous leishmaniasis: mucosal manifestations in an endemic country. Int J Infect Dis 2013; 17: e1088-9.

10.- McGwire B S, Satoskar A R. Leishmaniasis: clinical syndromes and treatment. QJM 2014; 107: 7-14.

11.- Soong L, Chang CH, Sun J, Longley B J Jr, Ruddle N H, Flavell R A, et al. Role of $\mathrm{CD} 4+\mathrm{T}$ cells in pathogenesis associated with Leishmania amazonensis infection. J Immunol 1997; 158: 5374-83.

12.- Alvar J, Aparicio P, Aseffa A, Den Boer M, Cañavate $\mathrm{C}$, Dedet JP, et al. The relationship between leishmaniasis and AIDS: the second 10 years. Clin Microbiol Rev 2008; 21 : 334-59.

13.- Ives A, Ronet C, Prevel F, Ruzzante G, Fuertes-Marraco S, Schutz F, et al. Leishmania RNA virus controls the severity of mucocutaneous leishmaniasis. Science 2011; 331: 775-8.

14.- Ronet C, Beverley S M, Fasel N. Muco-cutaneous leishmaniasis in the New World: the ultimate subversion. Virulence 2011; 2: 547-52.

15.- Tarqui K. Infección por virus linfotrópico de células T humanas I/II y su asociación con enfermedades parasitarias (estrongiloidiosis, leishmaniosis y tripanosomiosis). Rev Peru Epidemiol 2010; 14: 224-8

16.- Wood R. Clinical Features and Management of HIV/AIDS. Farrar J, Hotez P, Junghanss T, editors. Manson's Tropical Diseases. $23^{\text {rd }}$ ed. Saunders; 2014. pp: 79-96.

17.- Kopterides P, Mourtzoukou E G, Skopelitis E, Tsavaris N, Falagas M E. Aspects of the association between leishmaniasis and malignant disorders. Trans R Soc Trop Med Hyg 2007; 101: 1181-9.

18.- Amato V S, Nicodemo A C, Amato J G, Boulos M, Neto V A. Mucocutaneous leishmaniasis associated with HIV infection treated successfully with liposomal amphotericin B (AmBisome). J Antimicrob Chemother 2000; 46: 341-2.

19.- Padovese V, Terranova M, Toma L, Barnabas G A, Morrone A. Cutaneous and mucocutaneous leishmaniasis in Tigray, northern Ethiopia: clinical aspects and therapeutic concerns. Trans R Soc Trop Med Hyg 2009; 103: 707-11.

20.- Ronet C, Beverley S M, Fasel N. Un virus, hôte indésirable de L. guyanensis, détermine la gravité de la forme mucocutanée de la leishmaniose. Med Sci (Paris) 2011; 27: 924-6.

21.- Madeddu G, Fiori M L, Ena P, Riu F, Lovigu C, Nunnari G, et al. Mucocutaneous leishmaniasis as presentation of HIV infection in Sardinia, insular Italy. Parasitol Int 2014; 63: 35-6.

22.- Torrico F, Parrado R, Castro R, Marquez C J, Torrico M C, Solano M, et al. Co-infection of Leishmania (Viannia) braziliensis and HIV: report of a case of mucosal leishmaniasis in Cochabamba, Bolivia. Am J Trop Med Hyg 2009; 81: 555-8.

23.- Echevarría J, Campos P, Chang J, Cuéllar L, Gotuzzo E, Paz L, et al. Mucocutaneous leishmaniasis and AIDS: case report. Trans R Soc Trop Med Hyg 1993; 87: 186.

24.- Rodríguez-Morales A, Pascual-González Y, Benítez J, López-Zambrano M, Harter-Griep R, Vilca-Yengle L, et al. Asociación entre la incidencia de leishmaniosis cutánea y el índice de desarrollo humano y sus componentes en cuatro estados endémicos de Venezuela. Rev Peru Med Exp Salud Pública. 2010; 27 : 22-30.

25.- Lawn S D. Immune reconstitution disease associated with parasitic infections following initiation of antiretroviral therapy. Curr Opin Infect Dis 2007; 20: 482-8.

26.- Lindoso J A, Barbosa R N, Posada-Vergara M, Duarte M I, Oyafuso L K, Amato V S, et al. Unusual manifestations of tegumentary leishmaniasis in AIDS patients from the New World. Br J Dermatol 2009; 160: 311-8.

27.- David C V, Craft N. Cutaneous and mucocutaneous leishmaniasis. Dermatol Ther 2009; 22: 491-502.

28.- Pitarch G, Pitarch A. Úlcera nasal. Piel 2007; 22: 293-5.

29.- Boaventura V S, de Oliveira J G, Costa J M, Novais F O, de Oliveira C I, Barral-Netto M, et al. The value of the otorhinolaryngologic exam in correct mucocutaneous leishmaniasis diagnosis. Am J Trop Med Hyg 2009; 81: 384-6. 
30.- González M, Benito F, García L, Iglesias A. Leishmaniasis mucocutanea: una enfermedad importada con repercusión en ORL. Acta Otorrinolaringol Esp 2009; 60: 298-300.

31.- Herwaldt B L, Berman J D. Recommendations for treating leishmaniasis with sodium stibogluconate (Pentostam) and review of pertinent clinical studies. Am J Trop Med Hyg
1992; 46: 296-306.

32.- Tiuman T S, Santos A O, Ueda-Nakamura T, Dias Filho B P, Nakamura C V. Recent advances in leishmaniasis treatment. Int $\mathrm{J}$ Infect Dis 2011; 15: e525-32.

33.- Di Lella F, Vincenti V, Zennaro D, Afeltra A, Baldi A, Giordano D, et al. Mucocutaneous leishmaniasis: report of a case with massive involvement of nasal, pharyngeal and laryngeal mucosa. Int J Oral Maxillofac Surg 2006; 35: 870-2.

34.- González U, Pinart M, Rengifo-Pardo M, Macaya A, Alvar J, Tweed J A. Interventions for American cutaneous and mucocutaneous leishmaniasis. Cochrane Database Syst Rev 2009; (2): CD004834. 